\title{
Aggregation functions based on the Choquet integral applied to image resizing
}

\author{
Jessica C. S. Bueno ${ }^{a}$ and Camila Alves Dias ${ }^{a}$ and Graçaliz P. Dimuro ${ }^{a}$ and \\ Helida Santos ${ }^{a}$ and Eduardo N. Borges ${ }^{a}$ and Giancarlo Lucca ${ }^{a}$ Humberto Bustince $^{b}$ \\ ${ }^{a}$ Centro de Ciências Computacionais, Universidade Federal do Rio Grande, Rio Grande, Brazil, \\ jessica_bsaldivia@hotmail.com, cmdias@outlook.com.br, \{gracalizdimuro,helida,eduardoborges\}@ furg.br \\ ${ }^{b}$ Estadística, Informática y Matemáticas, Institute of Smart Cities, Universidad Pública de Navarra, \\ Pamplona, Spain, bustince@unavarra.es
}

\begin{abstract}
The rising volume of data and its high complexity has brought the need of developing increasingly efficient knowledge extraction techniques, which demands efficiency both in computational cost and in accuracy. Most of problems that are handled by these techniques has complex information to be identified. So, machine learning methods are frequently used, where a variety of functions can be applied in the different steps that are employed in their architecture. One of them is the use of aggregation functions aiming at resizing images. In this context, we introduce a study of aggregation functions based on the Choquet integral, whose main characteristic in comparison with other aggregation functions is that it considers, through fuzzy measure, the interaction between the elements to be aggregated. Thus, our main goal is to present an evaluation study of the performance of the standard Choquet integral the and copula-based generalization of the Choquet integral in relation to the maximum and mean functions, looking for results that may be better than the aggregation functions commonly applied. The results of such comparisons are promising, when evaluated through image quality metrics.
\end{abstract}

Keywords: Choquet integral, Aggregation functions, Image processing.

\section{Introduction}

Knowledge extraction techniques have received much attention from the research community in the past years, specially when one considers the rising volume of data and its increasing complexity. One of the most used techniques in the context of data extraction is classification [1], in which some instances have to be organized into predefined categories according to their features, and applied in various fields $[11,12,26,3]$, from health to emergency management, to name a few.

Image classification is a very common problem in the area of computer vision, where the main challenges are, for example: identifying patterns in images, distinguishing living beings from objects, labeling acquired images, among others. Most of these problems have complex information to be identified.

These methods use a variety of functions within the different steps that are employed in their architectures. One of them is the use of aggregation functions $[13,6]$, such as the maximum and the arithmetic mean [27].

Image reduction technique is commonly used to speed up processing or to reduce the storage or transmission cost (see, e.g., $[24,25,23,4])$. In order to improve the aggregation of meaningful information without degrading its discriminative power in image processing, Dias et al. [8] have proposed to replace the maximum and the arithmetic mean, which are commonly used in the literature for image reduction, by the Choquet integral [7]. In order to measure the output image quality, Dias et al. [8] considered the commonly used measurements found in the literature.

The main objective of this present work is to carry out a study of the use of an aggregation function defined as a copula-based generalization of the Choquet integral, called CC-integrals $[18,19]$, to reduce the size of an image. This study is performed by applying a particular instance of a CC-integral, called CMin-integral [18,9], to a group of images, obtaining the results of each image individually. The results are then compared with the Choquet Integral used in [8], the maximum and the arithmetic mean. Those comparisons of the results are performed through image quality measure functions [10].

We observe that CMin-integral has presented excellent results when used in classification problems the literature (see, e.g., $[18,9]$ ). CMin-integral was also used to build a multimodal fuzzy fusion-based brain-computer interface system [17]. Then, we are willing to evaluate if the CMinIntegral is able to present better results than the Choquet 
integral, the maximum and the mean functions.

This paper is organized as follows. In Section 2, we present some preliminary concepts, such as the definition of aggregation functions, as well as the definitions of Choquet integral and the generalisation of the Choquet integral based on copulae. In Section 3, the proposed method is addressed, where the process of resizing images by means of aggregation functions is detailed. The analysis of the experimental results are presented in Section 4. Finally, Section 5 presents the Conclusion.

\section{Preliminary concepts}

The process of combining different values and returning a single value is called aggregation. Formally, the operator responsible for this task is named an aggregation function [13]. The input values of an aggregation function can be, for example: membership degrees of fuzzy sets, weights of criteria, degrees of preference, etc. It can be applied in several areas such as statistics, computer science, mathematics and economics, to name a few.

Definition 1 An n-ary function $\mathcal{A}:[0,1]^{n} \rightarrow[0,1]$ is said to be an aggregation function if the following conditions hold:

(A1) Boundary conditions: $\mathcal{A}(0, \ldots, 0)=0$ and $\mathcal{A}(1, \ldots, 1)=1$.

(A2) Monotonicity: $\mathcal{A}\left(x_{1}, \ldots, x_{n}\right) \leq \mathcal{A}\left(y_{1}, \ldots, y_{n}\right)$ whenever $x_{i} \leq y_{i}$ for all $i \in\{1, \ldots, n\}$, i.e., $\mathcal{A}$ is nondecreasing in each argument.

The use of the domain $[0,1]^{n}$ and codomain $[0,1]$ to define the aggregation function aims the fuzzy context, but any closed interval could be used. The most commonly used aggregation functions are the maximum, defined by $f\left(x_{1}, \ldots, x_{n}\right)=\max \left\{x_{1}, \ldots, x_{n}\right\}$, the minimum, defined by $f\left(x_{1}, \ldots, x_{n}\right)=\min \left\{x_{1}, \ldots, x_{n}\right\}$, and the well known arithmetic mean function.

Definition 2 [16] An aggregation function $T:[0,1]^{2} \rightarrow$ $[0,1]$ is a t-norm if the following conditions hold, for all $x, y, z \in[0,1]$ :

(T1) Commutativity: $T(x, y)=T(y, x)$;

(T2) Associativity: $T(x, T(y, z))=T(T(x, y), z)$;

(T3) Neutral element 1: $T(x, 1)=x$.

One example of t-norm is the minimum defined by $T_{M}(x, y)=\min \{x, y\}$.

Copulas are important aggregation functions that link (twodimensional) probability distribution functions to their onedimensional marginal distributions, playing an important role in the theory of probabilistic metric spaces and statistics [2].
Definition 3 [2] A bivariate function $C:[0,1]^{2} \rightarrow[0,1]$ is a copula if it satisfies the following conditions, for all $x, x^{\prime}, y, y^{\prime} \in[0,1]$ with $x \leq x^{\prime}$ and $y \leq y^{\prime}$ :

(C1) $C[x, y]+C\left[x^{\prime}, y^{\prime}\right] \geq C\left[x, y^{\prime}\right]+C\left[x^{\prime}, y\right]$;

(C2) $C[x, 0]=C[0, x]=0$;

(C3) $C[x, 1]=C[1, x]=x$.

One example of copulas is the minimum t-norm $T_{M}$.

In this work we also consider the Choquet integral [5], which is an alternative aggregation function considered an extension of Lebesgue integral. It is defined based on a fuzzy measure, which can be interpreted in various ways depending on the context of the problem being addressed.

Within the context of aggregation functions, a fuzzy measure represents the degree of relation between the elements being aggregated [5]. Thus, the increasing use of Choquet integral occurs due to the fact that it considers the relevance of each attribute to be aggregated and also its interactions.

Definition 4 Let $N=\{1, \ldots, n\}$ and $2^{N}$ be the powerset of $N$. A function $\mathfrak{m}: 2^{N} \rightarrow[0,1]$ is a fuzzy measure if, for all $A, B \subseteq N$, the following conditions are satisfied:

(ml) Boundary conditions: $\mathfrak{m}(\emptyset)=0$ and $\mathfrak{m}(N)=1$.

(m2) Monotonicity: $\mathfrak{m}(A) \leq \mathfrak{m}(B)$, whenever $A \subseteq B$.

Definition 5 Let $\mathfrak{m}: 2^{N} \rightarrow[0,1]$ be a fuzzy measure. The discrete Choquet integral of $\vec{x}=\left(x_{1}, \ldots, x_{n}\right) \in[0,1]^{n}$ with respect to the fuzzy measure $\mathfrak{m}$ is the function $\mathfrak{C}_{\mathfrak{m}}$ : $[0,1]^{n} \rightarrow[0,1]$, defined by:

$$
\mathfrak{C}_{\mathfrak{m}}(\vec{x})=\sum_{i=1}^{n}\left(x_{(i)}-x_{(i-1)}\right) \cdot \mathfrak{m}\left(A_{(i)}\right),
$$

where $\left(x_{(1)}, \ldots, x_{(n)}\right)$ is an increasing permutation of $\vec{x}$, that is, $0 \leq x_{(1)} \leq \ldots \leq x_{(n)}$, where $x_{(0)}=0$, and $A_{(i)}=$ $\{(i), \ldots,(n)\}$ is the subset of indices corresponding to the $n-i+1$ largest components of $\vec{x}$.

The Choquet integral defined as in Eq. (1) satisfies the conditions of Def. 1, and therefore is an aggregation function. Besides, the Choquet integral can be written in its expanded form as:

$$
\mathfrak{C}_{\mathfrak{m}}(\vec{x})=\sum_{i=1}^{n}\left(x_{(i)} \cdot \mathfrak{m}\left(A_{(i)}\right)-x_{(i-1)} \cdot \mathfrak{m}\left(A_{(i)}\right)\right)
$$

Comparing with other aggregation functions, the main characteristic of the Choquet integral is that it takes into account, through the fuzzy measure, the interaction between the elements to be aggregated. For instance, the maximum does not consider the relation between the elements and 
disregards important information. In the context of image processing, the values to be aggregated are pixels that appear in a window. Therefore, in this sense, the more available information of the relations between the pixels to be aggregated, the better will be the output image.

Choquet integral can be generalized by copulas [2], yielding a family of aggregation functions called CC-integrals [18]. This generalization is constructed by replacing the product operator of the Choquet integral in its expanded form, Eq. (2), by a copula.

Definition 6 Let $\mathfrak{m}: 2^{N} \rightarrow[0,1]$ be a fuzzy measure and let $C:[0,1]^{2} \rightarrow[0,1]$ be a copula. A discrete Choquet integral based on copula with respect to the fuzzy measure $\mathfrak{m}$ is a function $\mathfrak{C}_{\mathfrak{m}}^{C}:[0,1]^{n} \rightarrow[0,1]$, defined by

$$
\mathfrak{C}_{\mathfrak{m}}^{C}(\vec{x})=\sum_{i=1}^{n} C\left(x_{(i)}, \mathfrak{m}\left(A_{(i)}\right)\right)-C\left(x_{(i-1)}, \mathfrak{m}\left(A_{(i)}\right)\right),
$$

where $\left(x_{(1)}, \ldots, x_{(n)}\right)$ is an increasing permutation of the input $\vec{x}$, i.e., $0 \leq x_{(1)} \leq \ldots \leq x_{(n)}$, with the convention that $x_{(0)}=0$, and $A_{(i)}=\{(i), \ldots,(n)\}$ is the subset of indices of the $n-i+1$ largest components of $\vec{x}$.

Note that Eq. (3) satisfies the conditions of Def. 1, and so $\mathfrak{C}_{\mathrm{m}}^{C}$ is also an aggregation function. Moreover, if we apply in Eq. (3) the minimum t-norm $T_{M}$, we obtain:

$\mathfrak{C}_{\mathfrak{m}}^{T_{M}}(\vec{x})=\sum_{i=1}^{n}\left(\min \left\{x_{(i)}, \mathfrak{m}\left(A_{(i)}\right)\right\}-\min \left\{x_{(i-1)}, \mathfrak{m}\left(A_{(i)}\right)\right\}\right)$,

which is known as the CMin-integral.

In this work we adopt as fuzzy measure, the power measure defined by:

$$
\mathfrak{m}_{P}(A)=\left(\frac{|A|}{n}\right)^{q}, \text { where } q>0 .
$$

The value of $q$ can be defined by an expert or be determined by a genetic algorithm which is able to determine the best value for $q$ according to the problem being addressed.[21, $20,18]$

\section{Proposed method}

It is shown in Figure 1 the process of input of an original image up to its modified output. It is depicted the resizing process of the image, i.e., a reduction by means of an aggregation function (in this case, the standard Choquet integral, Eq. (1) with the power measure as in Eq. (5), considering $q=0.5)$. As can see in in Figure 1 to carry out the process the input of a $4 \times 4$ image, regarding $2 \times 2$ windows, that is, 4 pixels by window with stride 2 .

Under this setting, the Choquet integral is applied on each one of the four image windows, generating a reduced image (a single value) in comparison with the input image, where now each of the four windows presents a pixel. In this way, the resulting aggregated image becomes a $2 \times 2$ image, where the yellow, green, blue and pink parts of the original image (reduced image by aggregation in Fig. 1) becomes single values.

Input: original image

\begin{tabular}{|c|c|c|c|}
\hline 66 & 32 & 76 & 82 \\
\hline 20 & 0 & 15 & 53 \\
\hline 15 & 241 & 230 & 147 \\
\hline 8 & 13 & 255 & 196 \\
\hline \multicolumn{4}{|c}{ Aggregation } \\
\hline
\end{tabular}

Reduced imaged by aggregation

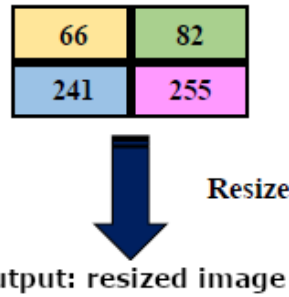

Output: resized image

\begin{tabular}{|c|c|c|c|}
\hline 66 & 66 & 82 & 82 \\
\hline 66 & 66 & 82 & 82 \\
\hline 241 & 241 & 255 & 255 \\
\hline 241 & 241 & 255 & 255 \\
\hline
\end{tabular}

Figure 1: Process steps: input and output of an image using maximum aggregation function

Then, in order to return to the same size of the input image, the resize step is applied after the aggregation step. The purpose of doing that is to make possible the use of quality measures to make comparisons between the input image with the output image, which needs to be into the same dimension. We used the nearest function in Matlab software. We observe that this resizing method is not the best one that may be found in the literature, such as image magnification using interval information [15]. However, since the resizing is not the focus of this work, we decided to use it due to its simplicity.

After this process, the quality measures can be applied. In this work we used seven of those [10], namely: $A v$ erage Difference (AD $\leq 0)$, Structural Content $(\mathrm{SC} \downarrow)$, Normalized Cross-Correlation (NK $\uparrow)$, Maximum Difference (MD $\downarrow$ ), Normalized Absolute Error (NAE $\downarrow$ ), Mean Squared Error (MSE $\downarrow$ ) and Peak Signal to Noise Ratio (PSNR $\uparrow$ ).

These measures aim at calculating up to what extent the output image is close/similar to the input image. The $\uparrow$ ar- 
row denotes that the higher the value, the better the quality. The $\downarrow$ arrow denotes that the lower the value, the better the quality of the output image.

Let an image be of size $M \times N$, where $M$ is the number of rows and $N$ the number of columns. Besides, let $P(i, j)$ represent the original image and let $\widehat{P}(i, j)$ represent the modified image (resized). The different quality metrics are defined as follows:

$$
\begin{aligned}
& A D=\frac{1}{M N} \sum_{i=1}^{M} \sum_{j=1}^{N}(P(i, j)-\widehat{P}(i, j)), \\
& S C=\frac{\sum_{i=1}^{M} \sum_{j=1}^{N}(P(i, j))^{2}}{\sum_{i=1}^{M} \sum_{j=1}^{N}(\widehat{P}(i, j))^{2}}, \\
& N K=\frac{\sum_{i=1}^{M} \sum_{j=1}^{N}(P(i, j) \times \widehat{P}(i, j))}{\sum_{i=1}^{M} \sum_{j=1}^{N}(P(i, j))^{2}}, \\
& M D=\max |P(i, j)-\widehat{P}(i, j)|, \\
& \text { for } i \in\{1, \ldots, M\} \text { and } j \in\{1, \ldots, N\} \text {. } \\
& N A E=\frac{\sum_{i=1}^{M} \sum_{j=1}^{N}|O(P(i, j))-O(\widehat{P}(i, j))|}{\sum_{i=1}^{M} \sum_{j=1}^{N}|O(P(i, j))|}, \\
& M S E=\frac{1}{M N} \sum_{i=1}^{M} \sum_{j=1}^{N}(P(i, j)-\widehat{P}(i, j))^{2}, \\
& P S N R=10 \log _{10} \frac{\left(2^{n}-1\right)^{2}}{\sqrt{M S E}},
\end{aligned}
$$

At last, quality measures are used and then hypothesis tests are applied in order to obtain a statistical analysis of the results. Non-parametric tests were used, since there is no guarantee of the normality and homocedasticity of data.

The non-parametric Friedman test [14] is applied to point out statistical differences between a group of results, that is, between the aggregation functions used. After confirming the existence of differences between groups, a post hoc test is performed to verify in which groups there are such differences. In order to do that, the Wilcoxon [28] nonparametric paired test was used. The significance level considered for the hypothesis tests was 0.05 .

\section{Results and discussion}

In Tables 2, 3, 4, 5, 6, 7 and 8 we find some results of each image quality measure. The table contains the results of the averages. The ones in blue color are the results for those functions based on Choquet integral, on the other hand in red color we have the results for functions commonly used in the literature and in boldface, the best aggregation functions of each type are given.

Twelve images were randomly chosen from IIIT $5 \mathrm{~K}$-Word dataset [22] in order to compare the following aggregation fuctions: maximum, arithmetic mean, standard Choquet integral and CMin-integral, Eq. (1) and Eq. (4), respectively, both using fuzzy power measure from Eq. (5).

For each of those twelve images, experiments were performed considering $2 \times 2,3 \times 3$ and $4 \times 4$ windows, and strides 2 and 3 . Using different sizes for windows and stride permits to capture interaction features between multiple windows. Besides, the $q$ parameter from Eq. (5) was varied considering the following values: $q=0.1, q=0.3$, $q=0.5$ and $q=0.7$.

In this way, each input image generated 24 output images, one for each distinct combination of the window settings, stride and exponent of the fuzzy measure. Then, seven quality measures were applied, namely: $\mathrm{AD} \leq 0, \mathrm{SC} \downarrow$, $\mathrm{NK} \uparrow, \mathrm{MD} \downarrow, \mathrm{NAE} \downarrow, \mathrm{MSE} \downarrow$ and PSNR $\uparrow$, which compared each output image with the input image.

Table 1 shows an extract of the complete data table of the measure $\mathrm{AD} \leq 0$, in order to illustrate how the data are organized to perform the analysis.

Table 1: Results for AD quality measure (result must be equal to or close to zero)

\begin{tabular}{c|c|c|c|c|c|c|c}
\hline Imagem & Stride & $\mathrm{q}$ & $\mathrm{W}$ & $\mathfrak{C}_{\mathfrak{m}}$ & $\mathfrak{C}_{\mathfrak{m}}^{\mathcal{T}_{M}}$ & Max & Mean \\
\hline 127_1 & 2 & 0.1 & 2 & -3.5849 & -6.8215 & -8.6169 & -0.1210 \\
$127 \_2$ & 2 & 0.1 & 2 & -4.2975 & -5.7222 & -11.4236 & -0.1309 \\
$127 \_3$ & 2 & 0.1 & 2 & -13.758 & -21.0464 & -6.4345 & -0.1191 \\
$138 \_4$ & 2 & 0.1 & 2 & -32.0959 & -41.1846 & -14.263 & -0.1245 \\
$138 \_5$ & 2 & 0.1 & 2 & -30.451 & -38.3909 & -16.3074 & -0.1319 \\
$138 \_6$ & 2 & 0.1 & 2 & -26.337 & -36.7698 & -15.1768 & -0.1313 \\
$159 \_1$ & 2 & 0.1 & 2 & 3.0724 & 22.473 & -20.9338 & -1.0202 \\
$161 \_1$ & 2 & 0.1 & 2 & -15.1256 & -9.4365 & -15.9019 & -0.1277 \\
$161 \_2$ & 2 & 0.1 & 2 & -13.2427 & -5.0373 & -15.4228 & -0.1258 \\
$52 \_1$ & 2 & 0.1 & 2 & -15.7669 & 4.5155 & -30.7347 & -0.4167 \\
$52 \_2$ & 2 & 0.1 & 2 & -27.9197 & -10.2939 & -33.6188 & -0.6948 \\
$52 \_3$ & 2 & 0.1 & 2 & -14.6652 & -4.7945 & -18.5452 & -1.1326 \\
\hline
\end{tabular}

It was possible to observe that the functions of maximum and integral aggregation of standard Choquet integral obtained better means for the AD $\leq 0$ quality measures Table (2), NK $\uparrow$ Table (6), SC $\downarrow$ Table (4) e MD $\downarrow$ Table (3). Since the null hypothesis of the Friedman test was rejected for quality measures, that is, there are statistical differences between the aggregation functions applied in each measure of quality.

The post-hoc test applied in each of measures quality AD 
$\leq 0, \mathrm{NK} \uparrow, \mathrm{SC} \downarrow$ and $\mathrm{MD} \downarrow$ indicated that only for $\mathrm{NK} \uparrow$ the maximum function and standard Choquet integral do not present significant differences. In view of this it can be concluded that, statistically, the two functions that obtained the best results for $\mathrm{AD}, \mathrm{NK} \uparrow, \mathrm{SC} \downarrow$ e $\mathrm{MD} \downarrow$ quality measures are different and presented superior results in relation to the mean and CMin-integral.

In the other side, for PSNR $\uparrow$ Table (8), NAE $\downarrow$ Table (5) and MSE $\downarrow$ Table (7) the means of each aggregation function showed the best results for the mean and CMin-Integral. Applying the Friedman test, the null hypothesis was rejected for the PSNR $\uparrow, N A E \downarrow$ and MSE $\downarrow$ measures. That is, there are also statistical differences between the aggregation functions for each of these measures. The Wilcoxon paired test applied to each of the PSNR $\uparrow$, NAE $\downarrow$ e MSE $\downarrow$ measures indicate that there are statistical differences between all aggregation functions for quality measures named $\mathrm{PSNR} \uparrow, \mathrm{NAE} \downarrow$ and MSE $\downarrow$.

It can be observed that for $\mathrm{AD} \leq 0, \mathrm{NK} \uparrow, \mathrm{SC} \downarrow$ and $\mathrm{MD} \downarrow$ measures the two best functions were the maximum and the standard Choquet integral. Alternatively, for PSNR $\uparrow$, $\mathrm{NAE} \downarrow$ and MSE $\downarrow$ measures the two best functions were the mean and CMin-Integral.

Table 2: Averages of the AD $\leq 0$ Image Quality Measurements for each aggregation function

\begin{tabular}{c|c|c|c}
\hline \multicolumn{4}{c}{$\mathrm{AD} \leq 0$} \\
\hline $\mathfrak{C}_{\mathfrak{m}}$ & $\mathfrak{C}_{\mathfrak{m}}^{T_{M}}$ & Max & Mean \\
\hline-23.0636 & $\mathbf{- 1 8 . 5 3 8 7}$ & -28.1740 & $\mathbf{1 . 4 9 3 0}$ \\
\hline
\end{tabular}

Table 3: Image Quality Measurement Averages MD $\downarrow$ for each aggregation function.

\begin{tabular}{c|c|c|c}
\hline \multicolumn{4}{|c}{$\mathrm{MD} \downarrow$} \\
\hline $\mathfrak{C}_{\mathfrak{m}}$ & $\mathfrak{C}_{\mathfrak{m}}^{T_{M}}$ & Max & Mean \\
\hline $\mathbf{1 6 4 . 9 4 4 4}$ & 188.4722 & $\mathbf{1 3 4 . 3 0 5 6}$ & 182.6667 \\
\hline
\end{tabular}

Table 4: Image Quality Measurement Means SC $\downarrow$ for each aggregation function.

\begin{tabular}{c|c|c|c}
\hline \multicolumn{4}{c}{$\mathrm{SC} \downarrow$} \\
\hline $\mathfrak{C}_{\mathfrak{m}}$ & $\mathfrak{C}_{\mathfrak{m}}^{T_{M}}$ & Max & Mean \\
\hline $\mathbf{0 . 7 4 7 7}$ & 0.8267 & $\mathbf{0 . 7 1 8 1}$ & 1.0961 \\
\hline
\end{tabular}

On the other hand, for the quality measures: PSNR $\uparrow, N A E \downarrow$ and MSE $\downarrow$, the averages of each aggregation function presented better results for the mean and CMin-Integral. Applying the Friedman test, the null hypothesis was rejected for PSNR $\uparrow, N A E \downarrow$ and MSE $\downarrow$ measures. Therefore, there are also statistical differences between the aggregation
Table 5: Averages of the Image Quality Measures NAE $\downarrow$ for each aggregation function.

\begin{tabular}{c|c|c|c}
\hline \multicolumn{4}{|c}{$\mathrm{NAE} \downarrow$} \\
\hline $\mathfrak{C}_{\mathfrak{m}}^{\mathfrak{c}}$ & $\mathfrak{C}_{\mathfrak{m}}^{T_{M}}$ & Max & Mean \\
\hline 0.3436 & $\mathbf{0 . 3 3 7 2}$ & 0.3489 & $\mathbf{0 . 1 9 9 0}$ \\
\hline
\end{tabular}

Table 6: Averages of Image Quality Measures NK $\uparrow$ for each aggregation function.

\begin{tabular}{c|c|c|c}
\hline \multicolumn{4}{c}{$\mathrm{NK} \uparrow$} \\
\hline $\mathfrak{C}_{\mathfrak{m}}$ & $\mathfrak{C}_{\mathfrak{m}}^{T_{M}}$ & Max & Mean \\
\hline $\mathbf{1 . 1 0 3 6}$ & 1.0627 & $\mathbf{1 . 1 1 6 7}$ & 0.9127 \\
\hline
\end{tabular}

Table 7: MSE $\downarrow$ Image Quality Measurement Means for each aggregation function.

\begin{tabular}{c|c|c|c}
\hline \multicolumn{4}{c}{ MSE $\downarrow$} \\
\hline $\mathfrak{C}_{\mathfrak{m}}$ & $\mathfrak{C}_{\mathfrak{m}}^{T_{M}}$ & Max & Mean \\
\hline 3069.48 & $\mathbf{2 5 8 1 . 1 0}$ & 4103.56 & $\mathbf{1 4 9 3 . 5 9}$ \\
\hline
\end{tabular}

Table 8: Averages of the Image Quality Measures PSNR $\downarrow$ for each aggregation function.

\begin{tabular}{c|c|c|c}
\hline \multicolumn{4}{c}{ PSNR $\downarrow$} \\
\hline $\mathfrak{C}_{\mathfrak{m}}$ & $\mathfrak{C}_{\mathfrak{m}}^{T_{M}}$ & Max & Mean \\
\hline 14.1419 & $\mathbf{1 4 . 5 3 4 4}$ & 13.2301 & $\mathbf{1 7 . 2 1 8 7}$ \\
\hline
\end{tabular}

functions for each of those quality measures. Wilcoxon paired test applied in each of the PSNR $\uparrow$, NAE $\downarrow$ and MSE $\downarrow$ measures, indicates that there are statistical differences between all the aggregation operators for PSNR $\uparrow, N A E \downarrow$ and $\mathrm{MSE} \downarrow$.

An interesting result is that, on one hand, it can be observed that for $\mathrm{AD} \leq 0, \mathrm{NK} \uparrow, \mathrm{SC} \downarrow$ and $\mathrm{MD} \downarrow$ the best functions were the maximum and standard Choquet integral. While for PSNR $\uparrow, N A E \downarrow$ and MSE $\downarrow$, the results were better for the mean and CMin-Integral.

Figures 3 and 4 show the original input image and output image after applying each of the aggregation functions considering $2 \times 2$ window, stride 2 and $q=0.5$. The values for the images, where it were applied the maximum and Choquet integral, in most cases were closer in comparison with the results from the mean and CMin-Integral, as seen in the tests. However, it is possible to verify visual differences on the integrity of the resulting images compared to the original input image.

In both Fig. 3 and Fig. 4 we observe that, for instance, the image of the maximum despite showing good results in the 


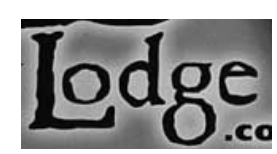

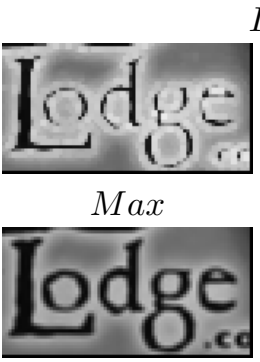

Mean
Input

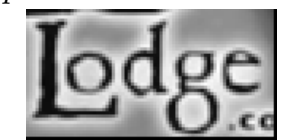

Choquet - integral

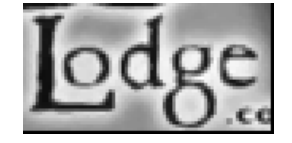

CMin-Integral
Figure 2: Maximum, mean, Choquet integral and CMinIntegral results obtained through experiments applied to one of the images from the IIIT $5 \mathrm{~K}$-Word dataset called 138_6 (after the resizing process). The parameters used were: window size $=4 \times 4$, stride $=2$ and exponent of the fuzzy measure $=0.7$

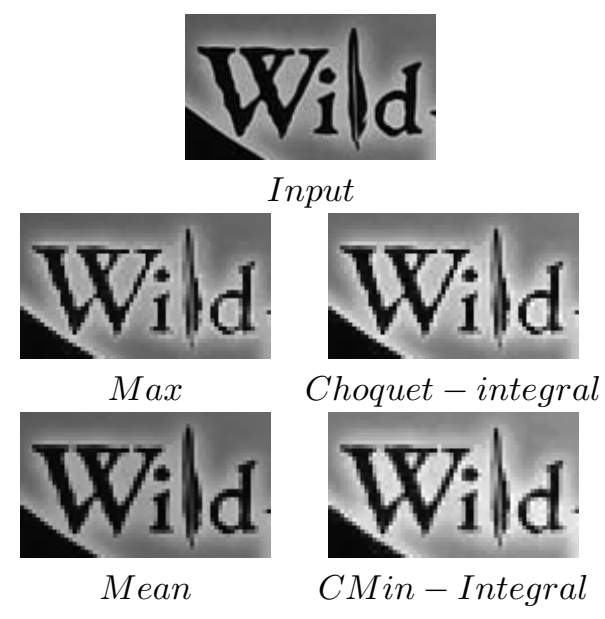

Figure 3: Maximum, mean, Choquet integral and CMinIntegral results obtained through experiments applied to one of the images from the IIIT 5K-Word dataset called 138 4 (after the resizing process). The parameters used were: window size $=2 \times 2$, stride $=2$ and exponent of the fuzzy measure $=0.1$

quality measures, is notably not so similar to the original image, as the edges are more jagged.

\section{Conclusion}

The process illustrated in Figure 1 is classic in the literature, only changing the sizes of windows and strides. The novelty of this paper was to present aggregation functions based on Choquet integral as an alternative from the classic ones, namely the maximum and mean functions.
The results of applying the Choquet integral to resizing images were shown to be good. It was possible to observe that the standard Choquet integral function has similar results to the maximum, and the CMin-Integral has similar results to the mean function. We also observed that Choquet integral aggregation function presented good quality of images maintaining the integrity of the original input image. That is, it was capable of minimizing the issues of jagged edges, despite not being the most similar to the original input image, as shown by the quality measures.

There is also the possibility of refining the results, in a way that the $q$ parameter used in the Choquet integral in relation to the fuzzy power measure can be learned by an algorithm, such as a neural network or a genetic algorithm, providing better results. This is still ongoing work.

Future works include also the use of other functions such as the pre-aggregation function presented in [21] and [20] in order to refine the choice of the $q$ parameter.

\section{Acknowledgement}

The authors Camila Dias and Jessica Bueno thank CAPES for the financial support received. Graçaliz P. Dimuro has funding from $\mathrm{CNPq} / \mathrm{Brazil}$ (process number 305882/20163). Eduardo N. Borges has funding from FAPERGS (TO 17/2551-0000872-3). Humberto Bustince is supported by the Spanish Ministry of Science and Technology (under project TIN2016-77356-P (AEI/FEDER, UE)).

\section{References}

[1] E. Alpaydin, Introduction to Machine Learning, 2nd Edition, The MIT Press, 2010.

[2] C. Alsina, B. Schweizer, M. J. Frank, Associative functions: triangular norms and copulas, World Scientific, 2006.

[3] G. Asencio-Cortés, F. Martínez-Álvarez, A. MoralesEsteban, J. Reyes, A sensitivity study of seismicity indicators in supervised learning to improve earthquake prediction, Knowledge-Based Systems 101 (2016) 15 -30 .

[4] G. Beliakov, H. Bustince, D. Paternain, Image reduction using means on discrete product lattices, IEEE Transactions on Image Processing 21 (3) (2012) 1070-1083.

[5] G. Beliakov, H. Bustince, T. C. Sánchez, A Practical Guide to Averaging Functions, Springer, 2016.

[6] G. Beliakov, A. Pradera, T. Calvo, Aggregation Functions: A Guide for Practitioners, Springer, Berlin, 2007.

[7] G. Choquet, Theory of capacities, Annales de 1'Institut Fourier 5 (1999) 131-295. 
[8] C. A. Dias, J. C. S. Bueno, E. N. Borges, S. S. C. Botelho, G. P. Dimuro, G. Lucca, J. Fernández, H. Bustince, P. L. J. D. Junior, Using the choquet integral in the pooling layer in deep learning networks, North american fuzzy information processing society annual conference 831 (2018) 144-154.

[9] G. P. Dimuro, G. Lucca, J. A. Sanz, H. Bustince, B. Bedregal, CMin-Integral: A Choquet-like aggregation function based on the minimum t-norm for applications to fuzzy rule-based classification systems, in: V. Torra, R. Mesiar, B. D. Baets (Eds.), Aggregation Functions in Theory and in Practice, Springer International Publishing, Cham, 2018, pp. 83-95.

[10] A. M. Eskicioglu, P. S. Fisher, Image quality measures and their performance, IEEE Transactions on communications 43 (12) (1995) 2959-2965.

[11] M. Galar, J. Derrac, D. Peralta, I. Triguero, D. Paternain, C. Lopez-Molina, S. García, J. M. Benítez, M. Pagola, E. Barrenechea, H. Bustince, F. Herrera, A survey of fingerprint classification part $\mathrm{i}$ : Taxonomies on feature extraction methods and learning models, Knowledge-Based Systems 81 (2015) 76 - 97.

[12] M. Galar, J. Derrac, D. Peralta, I. Triguero, D. Paternain, C. Lopez-Molina, S. García, J. M. Benítez, M. Pagola, E. Barrenechea, H. Bustince, F. Herrera, A survey of fingerprint classification part ii: Experimental analysis and ensemble proposal, KnowledgeBased Systems 81 (2015) 98 - 116.

[13] M. Grabisch, J. Marichal, R. Mesiar, E. Pap, Aggregation Functions, Cambridge University Press, Cambridge, 2009 .

[14] J. L. Hodges, E. L. Lehmann, Rank methods for combination of independent experiments in analysis of variance, The Annals of Mathematical Statistics 33 (2) (1962) 482-497.

[15] A. Jurio, M. Pagola, R. Mesiar, G. Beliakov, $\mathrm{H}$. Bustince, Image magnification using interval information, IEEE Transactions on Image Processing 20 (11) (2011) 3112-3123.

[16] E. P. Klement, R. Mesiar, E. Pap, Triangular Norms, Kluwer Academic Publisher, Dordrecht, 2000.

[17] L.-W. Ko, Y.-C. Lu, H. Bustince, Y.-C. Chang, Y. Chang, J. Ferandez, Y.-K. Wang, J. A. Sanz, G. P. Dimuro, C.-T. Lin, Multimodal fuzzy fusion for enhancing the motor-imagery-based brain computer interface, IEEE Computational Intelligence Magazine 14 (1) (2019) 96-106.

[18] G. Lucca, J. A. Sanz, G. P. Dimuro, B. Bedregal, M. J. Asiain, M. Elkano, H. Bustince, CC-integrals: Choquet-like copula-based aggregation functions and its application in fuzzy rule-based classification systems, Knowledge-Based Systems 119 (2017) 32-43.

[19] G. Lucca, J. A. Sanz, G. P. Dimuro, B. Bedregal, H. Bustince, A proposal for tuning the $\alpha$ parameter in $c_{\alpha} c$-integrals for application in fuzzy rule-based classification systems, Natural Computing (2018) 1-14.

[20] G. Lucca, J. A. Sanz, G. P. Dimuro, B. Bedregal, H. Bustince, R. Mesiar, CF-integrals: A new family of pre-aggregation functions with application to fuzzyrule-based classification systems., Information Sciences 435 (2018) 94-110.

[21] G. Lucca, J. A. Sanz, G. P. Dimuro, B. Bedregal, R. Mesiar, A. Kolesárová, H. Bustince, Preaggregation functions: Construction and an application., IEEE Transactions on Fuzzy Systems 24 (2) (2016) 260-272.

[22] A. Mishra, K. Alahari, C. Jawahar, Scene text recognition using higher order language priors, in: BMVCBritish Machine Vision Conference, BMVA Press, 2012, pp. 1-11.

[23] D. Paternain, H. Bustince, J. Fernandez, G. Beliakov, R. Mesiar, Image reduction with local reduction operators, in: International Conference on Fuzzy Systems, IEEE, 2010, pp. 1-8.

[24] D. Paternain, J. Fernández, H. Bustince, R. Mesiar, G. Beliakov, Construction of image reduction operators using averaging aggregation functions, Fuzzy Sets and Systems 261 (2015) 87-111.

[25] D. Paternain, A. Jurio, G. Beliakov, Color image reduction by minimizing penalty functions, in: 2012 IEEE International Conference on Fuzzy Systems, IEEE, 2012, pp. 1-7.

[26] S. Patidar, R. B. Pachori, U. R. Acharya, Automated diagnosis of coronary artery disease using tunableQ wavelet transform applied on heart rate signals, Knowledge-Based Systems 82 (2015) 1 - 10.

[27] D. Scherer, A. Müller, S. Behnkr, Evaluation of pooling operations in convolutional architectures for object recognition, International Conference on Artificial Neural Networks (2010) 92-101.

[28] F. Wilcoxon, Individual comparisons by ranking methods, Biometrics bulletin 1 (6) (1945) 80-83. 